EESTI NSV TEADUSTE AKADEEMIA TOIMETISED. FOOSIKA * MATEMAATIKA ИЗВЕСТИЯ АКАДЕМИИ НАУК ЭСТОНСКОП ССР ФИЗИКА * МАТЕМАТИКА PROCEEDINGS OF THE ACADEMY OF SCIENCES OF THE ESTONIAN SSR: PHYSICS * MATHEMATICS

$1984,33,1$

Г. АШКИНАЗИ, Э. ВЕЛМРЕ, А. ЛОГУСОВ, В. ТИМОФЕЕВ, Б. ФРЕИДИН, В. ШУМИЛИН

\title{
НЕИЗОТЕРМИЧЕСКАЯ ДИНАМИЧЕСКАЯ ПРЯМАЯ ВЕТВЬ ВОЛЬТ-АМПЕРНОЙ ХАРАКТЕРИСТИКИ СИЛОВЫХ АРСЕНИД-ГАЛЛИЕВЫХ ДИОДОВ
}

Предлагается универсальная физико-математическая модель для описания нестационарных электротепловых процессов в конструкции арсенид-галлиевого диода при протекании через него-мощного синусоидального импульса прямого тока длительностью 10 мс, проводится сопоставление расчетных и экспериментальных результатов.

\section{1. Исходные уравнения и метод решения}

Система дифференциальных уравнений, описывающая электротепловые процессы в полупроводниковой структуре, имеет в одномерном приближении следующий известный вид:

$$
\begin{aligned}
& \frac{\partial p}{\partial t}=-\frac{1}{q} \frac{\partial j_{p}}{\partial x}-R_{p}+G_{p} \\
& \frac{\partial n}{\partial t}=\frac{1}{q} \frac{\partial j_{n}}{\partial x}-R_{n}+G_{n} \\
& \frac{\partial E}{\partial t}=\frac{1}{\varepsilon \varepsilon_{0}}\left(j-j_{p}-j_{n}\right) \\
& \frac{\partial E}{\partial x}=\frac{q}{\varepsilon \varepsilon_{0}}(p-n+N) \\
& j_{p}=q \mu_{p} p E-q D_{p} \frac{\partial p}{\partial x} \\
& j_{n}=q \mu_{n} n E+q D_{n} \frac{\partial n}{\partial x} \\
& \varrho C_{p} \frac{\partial T}{\partial t}=\frac{\partial}{\partial x}\left(\lambda \frac{\partial T}{\partial x}\right)+Q .
\end{aligned}
$$

В системе использованы стандартные обозначе́ния, принятые в теории полупроводников: $n, p$ - концентрации электронов и дырок; $t$ - время; $q$ - элементарный заряд электрона; $x$ - координата; $j_{n}, j_{p}$ - плотности электронного и дырочного токов; $R_{n}, R_{p}, G_{n}, G_{p}-$ скорости рекомбинации и генерации электронов и дырок; $E-$ напряженность электрического поля; $\varepsilon_{0}-$ электрическая постоянная; $\boldsymbol{\varepsilon}$ - относительная диэлектрическая проницаемость; $N$ - концентрация основных носителей заряда; $\mu_{n}, \mu_{p}-$ подвижности электронов и дырок; 
$D_{n}, D_{p}-$ коэффициенты диффузии электронов и дырок; $\varrho$ - плотность; $C_{p}$ - удельная теплоемкость при постоянном давлении; $\lambda-$ коэффициент теплопроводности; $T$ - абсолютная температура; $Q-$ мощность рассеяния в единице объема.

Здесь (1), (2) - уравнения непрерывности для дырок и электронов соответственно; (3) - уравнение плотности полного тока; (4) - уравнение Пуассона; (5), (6) - уравнения плотностей дырочного и электронного токов соответственно; (7) - уравнение теплопроводности. В электротепловых процессах, происходящих в силовых полупроводниковых приборах при воздействии импульсов тока миллисекундного диапазона, характеристические постоянные времени электрических процессов намного меньше, чем тепловых, поэтому для искомых независимых переменных $p, n, E$ можно решать стационарную задачу, опустив в уравнениях (1) - (3) члены, содержащие производную по времени.

Общая скорость объемной рекомбинации, входящая в уравнения непрерывности (1), (2), представляет собой сумму скоростей рекомбинаций различных видов и определяется выражением

$$
R=R_{\mathrm{SRH}}+R_{A}+R_{\mathrm{u}}
$$

Скорость рекомбинации через локальные центры (рекомбинация Шокли-Рида-Холла) описывается выражением

$$
R_{S R H}=\frac{p n-n_{i}}{\tau_{p_{0}}\left(n+n_{1}\right)+\tau_{n_{0}}\left(p+p_{1}\right)}
$$

где $\tau_{p_{v}}, \tau_{n_{0}}, p_{1}, n_{1}$ - параметры модели Шокли-Рида-Холла. В настоящей работе принято, что $p_{1}=n_{1}=n_{i}$, где $n_{i}-$ собственная концентрация носителей заряда, температурная зависимость которой в арсениде галлия описывается формулой $\left[{ }^{2}\right]$

$$
n_{i}(T)=3,46 \cdot 10^{19}(T / 300)^{13 / 2} \exp (-9120 / T),
$$

которая дает $n_{i}(300 \mathrm{~K})=2,17 \cdot 10^{6} \mathrm{~cm}^{-3}$.

Температурная зависимость времен захвата $\tau_{n_{0}}, \tau_{p_{0}}$ взята из работы $\left[{ }^{3}\right]$

$$
\tau_{n_{0}, p_{0}}=\tau_{n_{0}, p_{0}}(300 \mathrm{~K})(T / 300)^{\boldsymbol{\alpha}_{\tau}},
$$

где использовались значения коэффициентов $\boldsymbol{\alpha}_{\tau}=+0,5$ для дырок и $\alpha_{\tau}=+1,4$ для электронов.

- Скорость межзонной Оже-рекомбинации определяется известным выражением

$$
R_{A}=\left(\gamma_{p} \cdot p+\gamma_{n} \cdot n\right)\left(p n-n_{i}^{2}\right),
$$

где $\gamma_{p}, \gamma_{n}-$ коэффициенты Оже-рекомбинации. В настоящей работе влияние Оже-рекомбинации $R_{A}$ не учитывалось.

Скорость межзонной излучательной рекомбинации описывается известным выражением

$$
R_{\mathbf{n}}=B\left(p n-n_{i}^{2}\right),
$$

где $B$ - коэффициент излучательной рекомбинации, температурная зависимость которого в случае достаточно чистого арсенида галлия имеет следующий теоретически установленный вид $\left[{ }^{4}\right]$ :

$$
B(T)=B(300 \mathrm{~K})(T / 300)^{-3 / 2}\left(E_{\mathrm{g}}(T) / E_{\mathrm{g}}(300 \mathrm{~K})\right)^{2} .
$$

Ввиду отсутствия данных по температурной зависимости $B(T)$ в сильнолегированном арсениде галлия в последнем случае также принята 
Значение параметров в слоях арсенид-галлиевой структуры

\begin{tabular}{|c|c|c|c|c|}
\hline \multirow{2}{*}{ Параметр } & \multicolumn{4}{|c|}{ Тип проводимости слоя } \\
\hline & $P^{+}$ & $P^{0}$ & $N^{0}$ & $N^{+}$ \\
\hline $\begin{array}{l}\text { Толщина слоя, мкм } \\
\text { Времена захвата }\end{array}$ & 50 & 91 & 54 & 45 \\
\hline $\begin{array}{l}\tau_{p_{0}}=\tau_{n_{0}}, \text { нс } \\
\text { Коэффициент излучательной } \\
\text { рекомбинащии, см }{ }^{3} / \mathrm{c}\end{array}$ & $\begin{array}{c}4 \\
2,0 \cdot 10^{-10} \\
{\left[{ }^{8}\right]}\end{array}$ & $\begin{array}{c}100 \\
7,21 \cdot 10^{-10} \\
{\left[{ }^{9}\right]}\end{array}$ & $\begin{array}{c}100 \\
7,21 \cdot 10^{-10}\end{array}$ & $\frac{4}{7,21 \cdot 10^{-10}}$ \\
\hline Подвижность электронов, см²/В·с & 400 & 5000 & $\begin{array}{l}\text { в виде } \\
\text { табл. 2 }\end{array}$ & 3000 \\
\hline Подвижность дырок, см²/B·c & 50 & 450 . & 450 & 200 \\
\hline
\end{tabular}

вышеуказанная зависимость $B(T)$.

Температурная зависимость ширины запрещенной зоны в арсениде галлия $E_{g}(T)$ учитывалась с помощью выражения [ $\left.{ }^{2}\right]$

$$
E_{g}(T)=E_{g_{0}}+\alpha_{g} T,
$$

где $E_{g_{0}}=1,575$ эВ; $\alpha_{g}=-5,0 \cdot 10^{-4}$ эВ $/ \mathrm{K}$.

В арсенид-галлиевых приборах наряду с межзонной излучательной рекомбинацией необходимо учитывать поглощение межзонного рекомбинационного излучения. Одним из способов учета скорости генерации электронно-дырочных пар за счет самопоглощения рекомбинационного излучения является подход, в соответствии с которым

$$
G=v \cdot R_{\text {й }}
$$

где $v-$ коэффициент фотонной генерации $\left[{ }^{1}\right]$, температурная зависимость которого может быть представлена следующей формулой $\left[{ }^{3}\right]$ :

$$
v(T)=v(300 \mathrm{~K}) \cdot(T / 300)^{\boldsymbol{a}_{v}},
$$

где $\alpha_{v}-$ эмпирический параметр, значение которого принято равным $-0,2$. $\left[{ }^{2}\right]$

Температурная зависимость подвижностей носителей заряда взята из

$$
\begin{aligned}
\therefore \quad \mu_{n}(T) & =\mu_{n}(300 \mathrm{~K})(T / 300)^{-2,3}, \\
\mu_{p}(T) & =\mu_{p}(300 \mathrm{~K})(T / 300)^{-2,5} .
\end{aligned}
$$

Связь между коэффициентами диффузии и подвижностями определялась соотношениями Эйнштейна. В настоящей работе рассеяние носителей на носителях не учитывалось.

таблица 2

Подвижность основных носителей заряда

в слаболегированной $N^{0}$-области

\begin{tabular}{c|c}
\hline, $\mathrm{cm}^{-3}$ & $\mu_{n}, \mathrm{~cm}^{2} / \mathrm{B} \cdot \mathrm{c}$ \\
\hline $1,0 \cdot 10^{15}$ & 6600 \\
$1,5 \cdot 10^{15}$ & 6500 \\
$2,2 \cdot 10^{15}$ & 6300 \\
$4,2 \cdot 10^{15}$ & 5800 \\
$1,0 \cdot 10^{16}$ & 5000 \\
$3,0 \cdot 10^{16}$ & 4200 \\
$7,3 \cdot 10^{16}$ & 3800 \\
$1,4 \cdot 10^{17}$ & 3000
\end{tabular}

Временная зависимость плотности полного тока $j(t)$, имеющая в общем случае произвольный характер, считалась заданной. Краевые условия для уравнений (1), (2) определялись из предположения омических контактов металл-полупроводник. В модели учитывались следующие источники тепла: распределенное выделение тепла в полупроводниковой структуре и сосредоточенное выделение джоулевого тепла в электрических контактных сопротивлениях между металлическими слоями. Считалось, что внутри металлических контактных слоев источ- 
Рис. 1. Распределение концентрации основных носителей заряда в различных слоях арсенидгаллиевой диодной структуры.

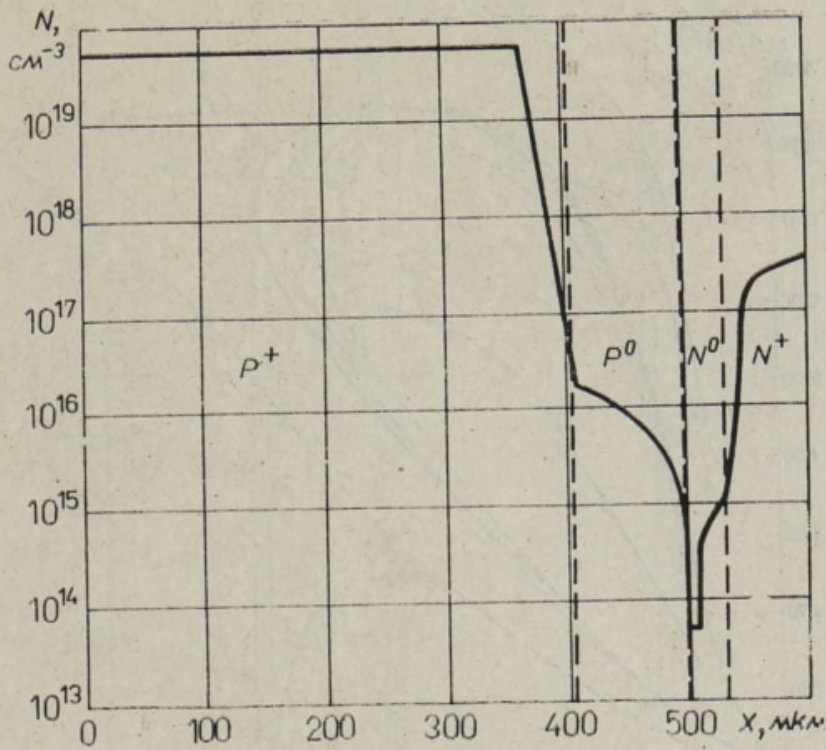

ники тепла отсутствуют. Мощность, выделяемая в полупроводнике, определяется главным образом двумя составляющими

$$
Q=j E+\left(R_{S R H}+R_{A}\right) \cdot E_{g} .
$$

Первая составляющая является джоулевой мощностью, а вторая учитывает мощность, передаваемую кристаллической решетке в результате рекомбинации электронов и дырок.

Температурные зависимости коэффициента теплопроводности $\lambda$ и удельной теплоемкости $C_{p}$ полупроводника описываются следующими формулами:

$$
\begin{gathered}
\lambda(T)=\lambda(300 \mathrm{~K})(T / 300)^{\alpha_{\lambda}}, \\
C_{p}(T)=C_{p_{o}}+\alpha_{c}(T / 300),
\end{gathered}
$$

где для арсенида галлия использовались значения параметров $\lambda_{i}(300 \mathrm{~K}):=0,425$ Вт $/$ смК, $\alpha_{\lambda}=-1,10$, приведенные в $\left[{ }^{2}\right]$, и $C_{p_{0}}=$ $=0,303$ Дж/г $\cdot \mathrm{K}$ и $\lambda_{\mathrm{c}}{ }^{\prime}=0,015$ Дж/г $/ \mathrm{K}\left[{ }^{2}\right]$.

Тепловые параметры металлов, входящих в контактные слои структуры, взяты из $\left[{ }^{5,6}\right]$. Ввиду слабой температурной зависимости коэффициенты теплопроводности и удельной теплоемкости металлических слоев в настоящей модели считались постоянными.

Для уравнения теплопроводности использовались следующие условия на границе раздела $i$-го и $i+1$-го слоев

$$
\begin{aligned}
& \lambda_{i} \frac{\partial T_{i}}{\partial x}=\lambda_{i+1} \frac{\partial T_{i+1}}{\partial x}+q_{i, i+1} \\
& T_{i}=T_{i+1}-R_{t_{t, t+1}} \lambda_{i+1} \frac{\partial T_{i+1}}{\partial x}
\end{aligned}
$$

где $R_{t, t+1}$ - тепловое контактное сопротивление. Поток джоулевого тепла $q_{i, i+1}$, выделяющегося в электрическом контактном сопротивлении $R_{k_{t, t+1}}$; описывается следующим выражением:

$$
q_{i, i+1}=j^{2} R_{k_{t, i+1}}
$$




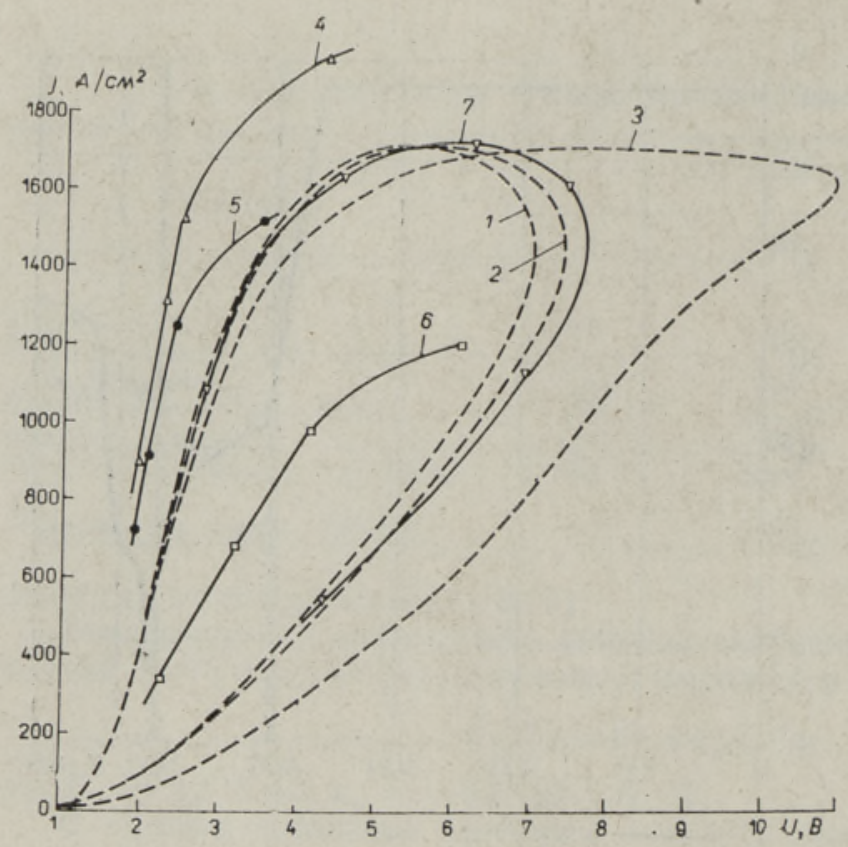

Рис. 2. Динамическая неизотермическая вольтамперная характеристика силовых арсенид-галлиевых днодов при воздействии синусоидального импульса тока длительностью 10 мс. $1,2,3-$ расчет (пунктирные линии): $j_{m}=1705 \mathrm{~A} / \mathrm{cm}^{2}$; $1-v=1,00 ; 2-v=$ $=0,99 ; \quad 3-v=0,99$ $(T / 300)^{-0,2} .4,5,6,7$ эксперимент (сплошные линии) при различных значениях $R_{д}, \mathrm{Oм} \cdot \mathrm{cm}^{2}: 4-$ $7,14 \cdot 10^{-4} ; 5-8,83 \cdot 10^{-4}$. $6-1,40 \cdot 10^{-3} ; 7$, $1,35 \cdot 10^{-3}$

В качестве начального условия для нестационарного теплового процесса принята одинаковая температура всех слоев структуры равная $T_{\text {гp }}$

$$
T_{i}(x, 0)=T_{\text {гр }}
$$

До воздействия мощного импульса тока выпрямительный элемент находился при комнатной температуре $\left(T_{\mathrm{rp}}=300 \mathrm{~K}\right)$ и через него протекал негреющий прямой ток плотностью $j=10 \mathrm{~A} / \mathrm{cm}^{2}$.

Нелинейная система исходных дифференциальных уравнений решается методом конечных разностей с помощью алгоритма, рассмотренного в $\left[{ }^{7}\right]$.

\section{2. Результаты расчета}

При моделировании рассматривалась следующая конструкция: слой 1 молибден (толщина 500 мкм), 2 - свинец (30 мкм), 3 - арсенид галлия (240 мкм), 4 - свинец (30 мкм), 5 - молибден (500 мкм), 6 - свинец (30 мкм), 7 - медь (3 мм).

Величины удельных контактных электрических и тепловых сопротивлений между различными слоями выпрямительного элемента, принятые в модели расчета, измерялись нами экспериментально и составили в среднем: $\quad R_{k_{1,2}}=R_{k_{4,5}}=R_{k_{5,0}}=R_{k_{0,7}}=5 \cdot 10^{-6} \quad \mathrm{Oм} \cdot \mathrm{cm}^{2} ; \quad R_{k_{2,3}}=$ $=1,2 \cdot 10^{-4} \mathrm{OM} \cdot \mathrm{cm}^{2} ; R_{k_{3,4}}=2,5 \cdot 10^{-4} \mathrm{OM} \cdot \mathrm{cm}^{2}$, а также $R_{t_{1,2}}=R_{t_{2,3}}=R_{t_{3,4}}=$ $=R_{t_{\mathrm{s}, 5}}=R_{t_{\mathrm{s}, \mathrm{s}}}=R_{t_{\mathrm{t}, \mathrm{7}}}=3,2 \cdot 10^{-2} \mathrm{Kcm}^{2} / \mathrm{BT}$.

Распределение концентрации примеси в арсенид-галлиевой диодной $P^{+}-P^{0}-N^{0}-N^{+}$-структуре изображено на рис. 1, значения основных параметров в слоях приведены в табл. 1 .

Взаимосвязь подвижности и концентрации основных носителей заряда в слаболегированной $N^{0}$-области охарактеризована в табл. 2.

Результаты моделирования неизотермических переходных процессов в арсенид-галлиевом силовом диоде при воздействии синусоидального импульса прямого тока с амплитудой $j_{m}=1705 \mathrm{~A} / \mathrm{cm}^{2}$ и длительностью 10 мс приведены на рис. 2. Одномерное температурное поле арсенид-гал- 


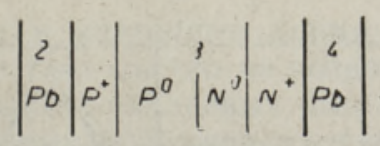

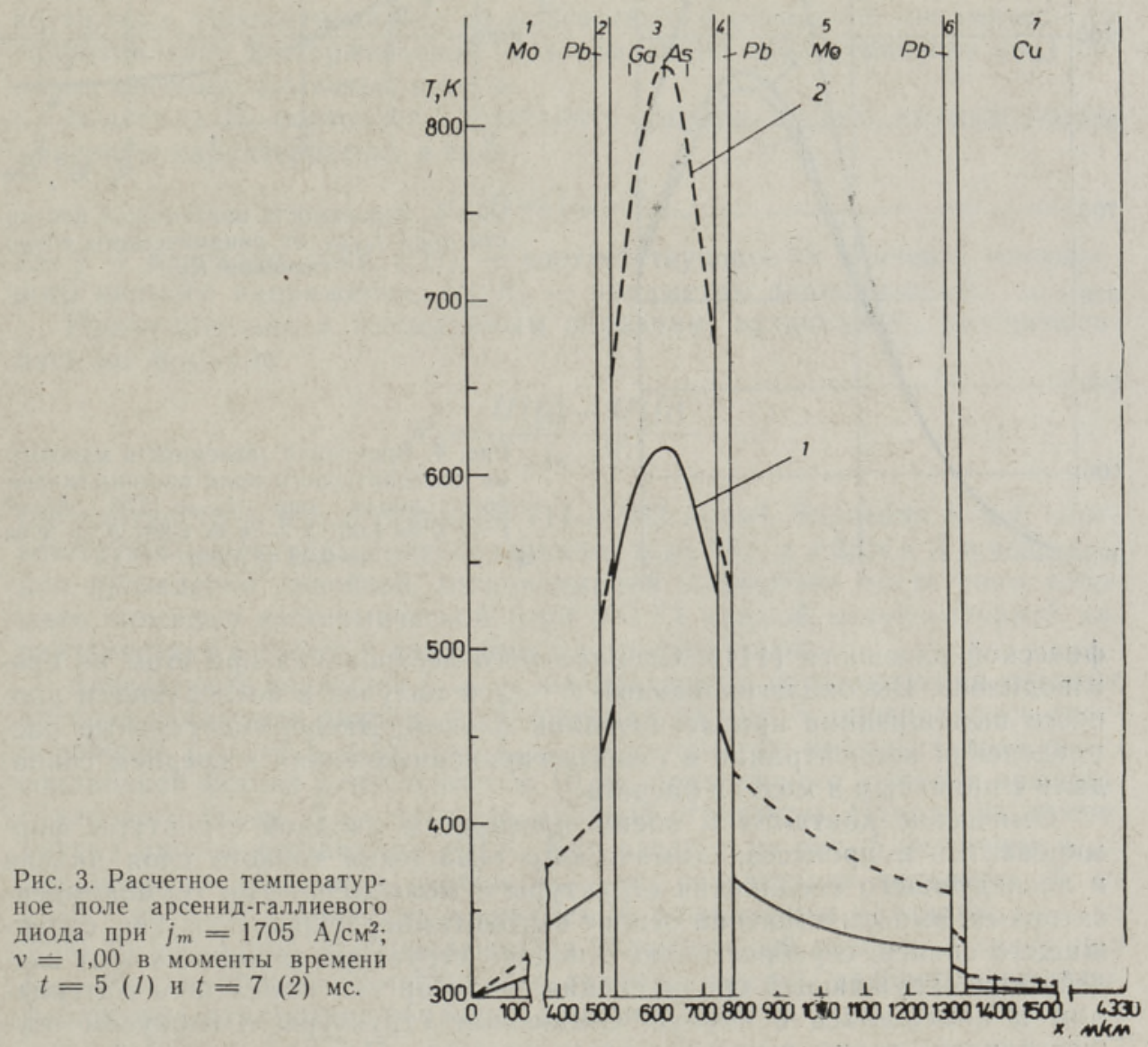

лиевого диода в моменты времени $t=5$ и 7 мс показано на рис. 3 , временная зависимость максимума температурного поля диода - на рис. 4 .

Все расчеты проводились на ЭВМ ЕС-1022. Количество узлов в пространственной сетке составляло 193 (из них 82 в арсениде галлия). Типичное время счета неизотермической вольт-амперной характеристики в интервале времени до 10 мс с равномерным временным шагом 0,5 мс составляло при одном значении амплитуды тока $\sim 45$ мин.

\section{3. Экспериментальное исследование неизотермической прямой вольт-амперной характеристики арсенид-галлиевых диодов}

Экспериментально исследуемые образцы представляли собой макеты силовых арсенид-галлиевых диодов на токи 25-40 А с напряжением пробоя $200-300$ В.

Основой исследуемых образцов является арсенид-галлиевая диодная структура типа $P+-P^{0}-N^{0}-N^{+}$, слаболегированные слои $P^{0}, N^{0}$ которой получены в едином процессе выращивания из ограниченного объема раствора-расплава на подложках $P^{+}$-типа, легированных цинком до уровня $\sim 5 \cdot 10^{19} \mathrm{~cm}^{-3}$ и ориентированных в направлении кристаллогра- 

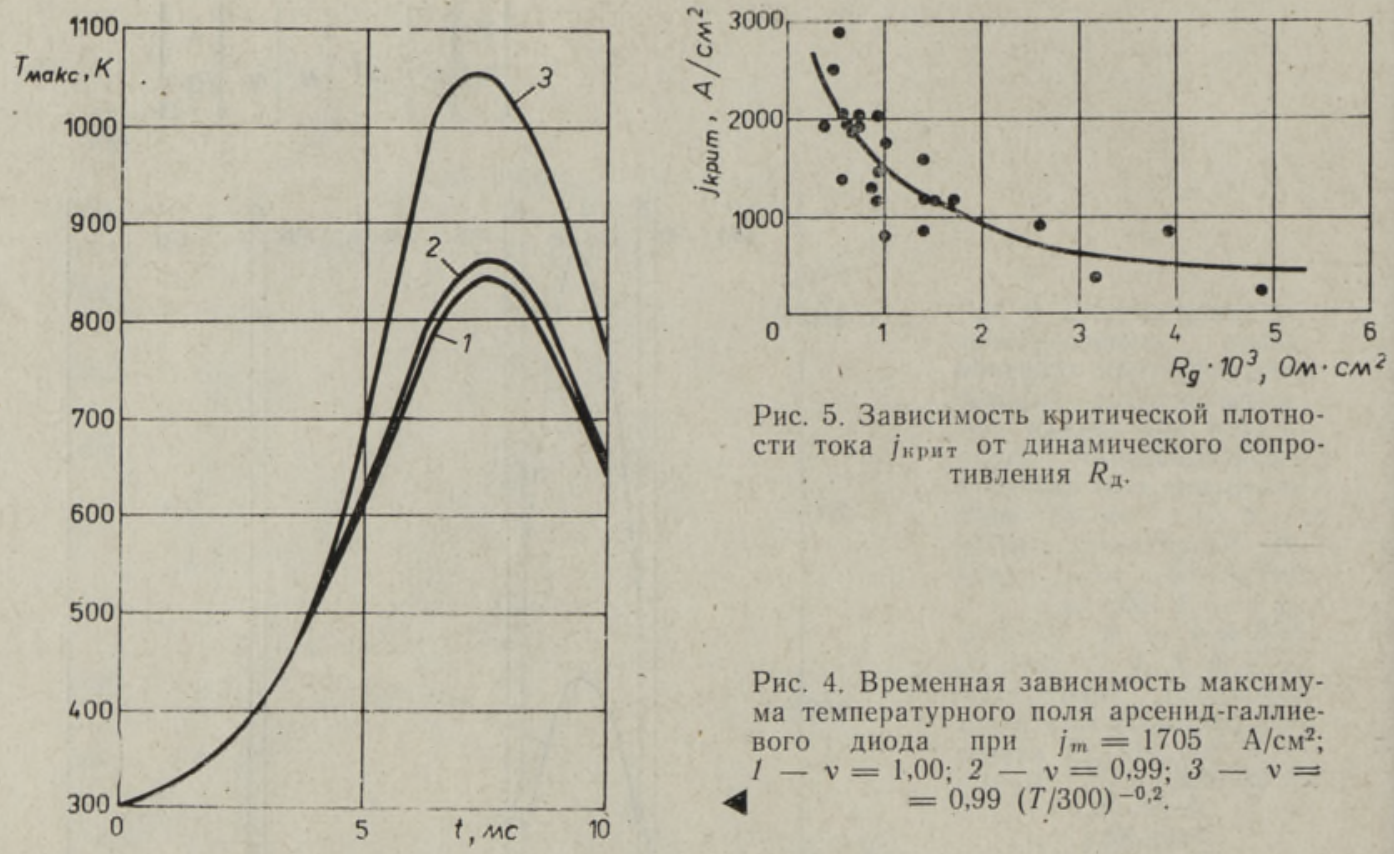

Рис. 5. Зависимость критической плотности тока $j_{\text {крит }}$ от динамического сопротивления $R_{д}$.

Рис. 4. Временная зависимость максимума температурного поля арсенид-галлие вого диода при $j_{m}=1705 \mathrm{~A} / \mathrm{cm}^{2}$; $1-v=1,00 ; 2-v=0,99 ; 3-v=$ $4 \quad=0,99(T / 300)^{-0,2}$.

фической плоскости (111). Специального легирования при этом не производилось. Высоколегированный $N^{+}$-слой получен в последующем процессе выращивания при легировании оловом. Экспериментальные распределения концентрации в слаболегированных слоях в среднем совпадали с принятым в модели расчета.

Омические контакты к обеим плоскостям диодной структуры формировались в процессах химического осаждения тонкого слоя никеля и последующего соединения структуры с молибденовыми термокомпенсаторами, выполняемого на основе высокотемпературного сплава, содержащего свинец, серебро, олово. Диодная структура защищалась от воздействия окружающей среды специальным органосиликатным материалом и помещалась в корпус. Соединение структуры с корпусом осуществлялось также вышеуказанным сплавом. Площадь диодной структуры составляла $0,35 \mathrm{~cm}^{2}$.

Проведены измерения динамической неизотермической прямой вольтамперной характеристики силовых арсенид-галлиевых диодов при воздействии на них однократного синусоидального греющего импульса тока длительностью 10 мс и при начальной температуре исследуемых диодов 300 K. Сравнение экспериментальных (4-7) и расчетных (1 и 2) кривых показывает их совпадение в пределах погрешности эксперимента и принятых при расчете допущений (рис. 2). При этом, как следует из расчетных зависимостей $(1,2)$, изменение величины $v$ от 0,99 до 1,00 не оказывает существенного влияния на динамическую неизотермическую вольт-амперную характеристику арсенид-галлиевых диодов с указанными выше электрофизическими параметрами.

Важнейшим практическим применением предложенной физико-математической модели является прогнозирование стойкости моделируемых диодов к воздействию токов ударных перегрузок. Известно, что причиной параметрического отказа диода в режиме ударной перегрузки является мгновенное превышение максимальной температуры полупроводниковой структуры выше критической $\left[{ }^{10}\right]$. Приведенные на рис. $2-4$ экспериментальные зависимости позволяют найти соответствующую критической температуре величину критической плотности тока. Необходимым условием при этом, очевидно, является предварительное определение 
величины критической температуры, которая в настоящее время для арсенид-галлиевых диодов неизвестна. Это обстоятельство не позволяет провести теоретическую оценку стойкости арсенид-галлиевых диодов к воздействию токов ударных перегрузок. Поэтому в рамках настоящей работы проводилось экспериментальное определение критической плотности тока, рассматривалась ее взаимосвязь с величиной динамического сопротивления изотермической вольт-амперной характеристики $\left(R_{д}\right)$ силовых арсенид-галлиевых диодов.

Величина $R_{д}$ позволяет представить нзотермическую прямую вольтамперную характеристику в виде:

$$
U(j)=U_{0}+R_{\text {z }},
$$

где $j$ - плотность тока, $U(j)$ - соответствующее ей значение импульсного прямого напряжения, $U_{0}, R_{д}$ - параметры линеаризации.

Применительно к исследуемым образцам, величина $R_{\text {д }}$ рассчитывалась по формуле

$$
R_{\text {д }}=\frac{U\left(j_{2}\right)-U\left(j_{1}\right)}{j_{2}-j_{1}},
$$

где плотности $j_{2}$ и $j_{1}$ составляли 714 и $223 \mathrm{~A} / \mathrm{cm}^{2}$. Значения $j_{2}$ и $j_{1}$ соответствуют амплитудам синусоидального тока 80 Х $л$ и $25 \times \pi$ А и выбраны для проведения линейной аппроксимации вследствие достаточно хорошего описания изотермической (при $300 \mathrm{~K}$ ) прямой вольт-амперной характеристики в рабочем диапазоне плотностей токов.

Зависимость величины $j_{\text {крит }}$ от динамического сопротивления силовых арсенид-галлиевых диодов, изображенная на рис. 5 , определялась соотношением $j_{\text {крит }}=I_{\text {крит }} / S$, где $I_{\text {крит }}$ - амплитуда импульса тока синусоидальной формы длительностью 10 мс, приводящего к отказу арсенидгаллиевого диода, $S$ - площадь диодной структуры. Начальная температура диодов составляла $300 \mathrm{~K}$. Как следует из рис. 5 , величина $j_{\text {крит }}$ закономерно убывает с ростом $R_{д}$. Так, возрастание величины $R_{д}$ от

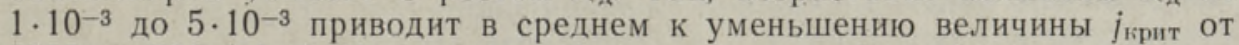
2500 до $500 \mathrm{~A} / \mathrm{cm}^{2}$. Наблюдающийся значительный разброс в значениях

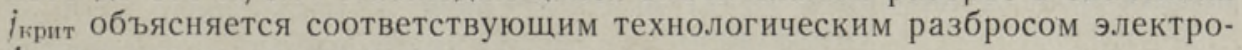
физических параметров реальных диодов.

\section{ЛИТ Е РА Т У РА}

1. Алферов Ж. И., Аикинази Г. А., Корольков В. Н., Падьюс А. А., Тимофеев В. Н., Челноков В. Е., Шумилин В. Н. ФТП, 12, вып. 7, 1336-1341 (1978).

2. Mayer, J. R., Kruer, M. R., Bartoli, F. J. J. Appl. Phys., 51, № 10, 5513-5522 (1980).

3. Аикинази Г. А., Киви У. М., Тимофеев В. Н. ФТП, 15, вып. 4, 718-724 (1981).

4. Мосс Т., Баррел Г., Эллис Б. Полупроводниковая оптоэлектроника. М., «Мир», 1976.

5. Таблицы физических величнн. Справочник. (Под ред. И. К. Кикоина.) М., Атомнздат, 1976.

6. Уонг X. Основные формулы и данные по теплообмену для инженеров. Справочник. М., Атомиздат, 1979.

7. Велмре Э. Э., Фрейдин Б. П. Электронное моделирование, 1983, № 1, 73-76.

8. Casey, Jr. H. C. Stern, F. J. Appl. Phys., 1976, 47, № 2, 631-641.

9. Varcchni, Y. P. Phys. Stat. Solidi. 1967, 19, 459.

10. Рабинерсон А. А., Ашкинази Г. А. Режимы нагрузки силовых полупроводниковых приборов. М., «Энергия», 1976.

Научно-исследовательский институт

Таллинского электротехнического завода им. М. Н. Калинина

Таллинский политехнический институт 


\section{GALLIUMARSENIIDIST JOUDIOODIDE MITTEISOTERMILINE DUNAAMILINE PARIVOOLU VOLTAMPERKARAKTERISTIK}

Galliumarseniidist jõudioodide konstruktsioonielementides toimuvate mittestatsionaarsete elektriliste ja soojuslike protsesside kirjeldamiseks on esitatud universaalne füüsikalismatemaatiline mudel, mis põhineb pooljuhtide fenomenoloogiliste võrrandite süsteemi ja soojusjuhtivuse võrrandi lahendamisel. On arvestatud mittekiirguslikku ia kiirguslikku rekombinatsiooni ja rekombinatsioonikiirguse iseneeldumisest tingitud elektron-auk paaride generatsiooni ning kontaktide elektrilisi ia soojustakistusi.

On uuritud elektrilisi ja soojusnähtusi dioodis, mida läbib ühekordne sinusoidaalne vooluimpulss (amplituud $1700 \mathrm{~A} / \mathrm{cm}^{2}$, impulsi kestus $10 \mathrm{~ms}$ ). Mitteisotermilise dünaamilise pärivoolu voltamperkarakteristiku mōōtmistulemuste vōrdlemisel arvutustulemustega on täheldatud kokkulangemist eksperimendi ja mudeli täpsuse piirides.

G. ASHKINAZI, E. VELMRE, A. LOGUSOV,

V. TIMOFEYEV, B. FREIDIN, V. SHUMILIN

\section{NONISOTHERMAL DYNAMIC FORWARD CURRENT-VOLTAGE CHARACTERISTICS OF GALLIUM ARSENIDE POWER DIODES}

A universal model of nonequilibrium electrical and thermal processes in gallium arsenide power diodes is proposed. The model is based on a numerical solution of phenomenological equations of electrical and thermal conductivity in semiconductors. The model takes into account nonradiative and radiative mechanisms of recombination, generation of electron-hole pairs by radiation, and electrical and thermal contact resistances. Forward current-voltage characteristics and thermal fields of a gallium arsenide power diode with sine-current pulse are calculated. Current pulse amplitude was about $1700 \mathrm{~A} / \mathrm{cm}^{2}$ and it was $10 \mathrm{~ms}$ long. Experimental and calculated results coincide quite satisfactorily. 